\title{
ASYMPTOTIC RESULTS FOR WEIGHTED MEANS OF RANDOM VARIABLES WHICH CONVERGE TO A DICKMAN DISTRIBUTION, AND SOME NUMBER THEORETICAL APPLICATIONS
}

\author{
Rita Giuliano $^{1}$ And Claudio $\mathrm{MACCI}^{2}$
}

\begin{abstract}
This paper studies some examples of weighted means of random variables. These weighted means generalize the logarithmic means. We consider different kinds of random variables and we prove that they converge weakly to a Dickman distribution; this extends some known results in the literature. In some cases we have interesting connections with number theory. Moreover we prove large deviation principles and, arguing as in [R. Giuliano and C. Macci, J. Math. Anal. Appl. 378 (2011) 555-570], we illustrate how the rate function can be expressed in terms of the Hellinger distance with respect to the (weak) limit, i.e. the Dickman distribution.
\end{abstract}

Mathematics Subject Classification. 60F10, 60F05, 11K99.

Received January 13, 2014. Revised August 5, 2014.

\section{INTRODUCTION}

In this paper we prove large deviation principles for some examples of weighted means $\left\{Z_{n}: n \geq 1\right\}$ defined by

$$
Z_{n}:=\frac{1}{L(n)} \sum_{k=1}^{n} \frac{\phi(k)-\phi(k-1)}{\phi(k)} W_{k},
$$

where $\left\{W_{n}: n \geq 1\right\}$ is a sequence of real valued random variables, $\phi: \mathbb{N} \rightarrow[0, \infty)$ is a strictly increasing function such that

$$
\phi(0)=0, \lim _{n \rightarrow \infty} \phi(n)=\infty, \text { and } \lim _{n \rightarrow \infty} \frac{\phi(n)}{\phi(n+1)}=1,
$$

and $L(n)=L_{\phi}(n)$ is defined by

$$
L(n):=\sum_{k=1}^{n} \frac{\phi(k)-\phi(k-1)}{\phi(k)} .
$$

Keywords and phrases. Almost sure central limit theorem, Dickman function, Hellinger distance, large deviations, prime numbers, square-free numbers.

1 Dipartimento di Matematica, Università di Pisa, Largo Bruno Pontecorvo 5, 56127 Pisa, Italy. giuliano@dm.unipi.it

2 Dipartimento di Matematica, Università di Roma Tor Vergata, Via della Ricerca Scientifica, 00133 Rome, Italy.

macci@mat.uniroma2.it 
In particular we consider logarithmic means, i.e. the case in which

$$
\phi(n):=n(\text { for all } n \geq 1)
$$

the term "logarithmic" comes from the equivalence relation $L(n)=\sum_{k=1}^{n} \frac{1}{n} \sim \log n$ (throughout the paper we write $x_{n} \sim y_{n}($ as $n \rightarrow \infty)$ to mean $\left.\lim _{n \rightarrow \infty} \frac{x_{n}}{y_{n}}=1\right)$. Logarithmic means have a relationship with the empirical measures which appear in the almost sure limit theorems in the literature. The most famous almost sure limit theorem is the so-called almost sure central limit theorem which was proved independently in $[4,13,32]$ under strong moment assumptions; successive refinements appear in [14,25], in which only finite variance is required. A survey on the almost sure central limit theorem and some developments can be found in [2]. Wider classes of almost sure limit theorems are presented in [3,22]; other different almost sure limits related to the extreme value theory can be found in $[6,12]$ (a more general result in this direction is proved in [11]).

Large deviations give an asymptotic computation of small probabilities on an exponential scale (see e.g. [8] as a reference on this topic). Large deviation results for empirical measures associated to almost sure central limit theorems can be found in $[19,30]$; in those references all the (common) moments of the random variables are finite, and the optimality of the moment assumptions is discussed in [28]. We also recall the large deviation results in [31] for the so-called Lévy strong arc-sine law (see [27]), and in [24] for a suitable class of weighted means.

Now we briefly recall some basic preliminaries on large deviations (see e.g. [8], p. 4 and 5). Let $\mathcal{X}$ be a topological space equipped with its completed Borel $\sigma$-field. A sequence of $\mathcal{X}$-valued random variables $\left\{Z_{n}\right.$ : $n \geq 1$ \} satisfies the large deviation principle (LDP for short) with speed function $v_{n}$ and rate function $I$ if: $\lim _{n \rightarrow \infty} v_{n}=\infty$; the function $I: \mathcal{X} \rightarrow[0, \infty]$ is lower semi-continuous; we have the upper bound

$$
\limsup _{n \rightarrow \infty} \frac{1}{v_{n}} \log P\left(Z_{n} \in F\right) \leq-\inf _{x \in F} I(x) \text { for all closed sets } F,
$$

and the lower bound

$$
\liminf _{n \rightarrow \infty} \frac{1}{v_{n}} \log P\left(Z_{n} \in G\right) \geq-\inf _{x \in G} I(x) \text { for all open sets } G .
$$

A rate function $I$ is said to be good if its level sets $\{\{x \in \mathcal{X}: I(x) \leq \eta\}: \eta \geq 0\}$ are compact. In the LDPs presented in this paper we always have $\mathcal{X}=\mathbb{R}$; moreover we often apply the Gärtner-Ellis's Theorem (see e.g. Thm. 2.3.6 in [8]), and the rate functions are good.

In all the LDPs in this paper (except one in the final section which gives a counterexample) the random variables $\left\{Z_{n}: n \geq 1\right\}$ in (1.1) are defined by setting

$$
W_{n}:=\frac{1}{\phi(n)} \sum_{k=1}^{n} \phi(k) R_{k}
$$

for suitable independent random variables $\left\{R_{n}: n \geq 1\right\}$, and we prove that $\left\{W_{n}: n \geq 1\right\}$ converges weakly to a Dickman distribution; moreover the sequence $\left\{R_{n}: n \geq 1\right\}$ depends on a sequence of positive numbers $\left\{\lambda_{n}: n \geq 1\right\}$ such that

$$
\lim _{n \rightarrow \infty} \frac{1}{\phi(n)} \sum_{k=1}^{n} \phi(k) \lambda_{k}=\nu, \text { for some } \nu \in(0, \infty),
$$

and the common rate function is

$$
I(x):= \begin{cases}x \log \frac{x}{\nu}-x+\nu & \text { if } x \geq 0 \\ \infty & \text { if } x<0\end{cases}
$$

where $0 \log 0=0$. 
Remark 1.1. By (1.2), the limit relation (1.5) always holds (with $\nu=1$ ) if we choose the sequence $\left\{\lambda_{n}: n \geq 1\right\}$ defined by $\lambda_{n}:=\frac{\phi(n)-\phi(n-1)}{\phi(n)}$ for all $n \geq 1$.

The weak convergence results for the sequences $\left\{W_{n}: n \geq 1\right\}$ presented in this paper provide extensions of some results in the literature (more precisely in $[1,5]$ ). Moreover a LDP presented in this paper is obtained by combining other LDPs and a standard large deviation technique which is based on the concept of exponential equivalence. All these results depend only on the asymptotic behavior of the function $\phi$ and of the sequence $\left\{\lambda_{n}: n \geq 1\right\}$ in (1.2) and (1.5).

As it happens for the LDPs in [16], the rate function in (1.6) can be expressed in terms of condition $(\mathbf{H})$ (see Sect. 5 in this paper), i.e. a suitable formula with the Hellinger distance with respect to the weak limit $P_{W_{\infty}}$ of the sequence $\left\{W_{n}: n \geq 1\right\}$. Actually we also present the LDP for a sequence of logarithmic means $\left\{Z_{n}: n \geq 1\right\}$ where the rate function does not meet condition $(\mathbf{H})$; this might be explained noting that the sequence $\left\{W_{n}: n \geq 1\right\}$ in this example is not a sequence of partial sums of random variables forming a triangular array (as it happens for the other examples in this paper, and for the ones in [16]).

Throughout the paper we use the following symbols when a random variable $Z$ is Dickman, Poisson, Bernoulli or exponential distributed, respectively.

- $Z \stackrel{d}{\sim} \mathcal{D}(\nu)$, for $\nu>0$, if

$$
\mathbb{E}\left[\mathrm{e}^{s Z}\right]=\exp \left(\nu \int_{0}^{s} \frac{\mathrm{e}^{y}-1}{y} \mathrm{~d} y\right)=\exp \left(\nu \int_{0}^{1} \frac{\mathrm{e}^{s y}-1}{y} \mathrm{~d} y\right) \text { for all } s \in \mathbb{R} .
$$

- $Z \stackrel{d}{\sim} \mathcal{P}(\nu)$, for $\nu>0$, if $Z$ is a random variable such that $P(Z=k)=\frac{\nu^{k}}{k !} \mathrm{e}^{-\nu}$ for all integer $k \geq 0$.

- $Z \stackrel{d}{\sim} \mathcal{B}(p)$, for $p \in[0,1]$, if $Z$ is a random variable such that $P(Z=1)=1-P(Z=0)=p$.

- $Z \stackrel{d}{\sim} \mathcal{E}(\nu)$, for $\nu>0$, if $Z$ is a random variable such that $P(Z \leq t)=1-\mathrm{e}^{-\nu t}$ for all $t \geq 0$.

The distribution $\mathcal{D}(\nu)$ is related to the Dickman's function (see e.g. [34], Sect. III.5.4). The Dickman's function $\rho(x)$ is defined as the continuous solution of the delay differential equation

$$
u \rho^{\prime}(u)+\rho(u-1)=0(u>1)
$$

with the initial condition $\rho(u)=0$ for $u \in[0,1]$. This function plays an important role in analytic number theory: see the fundamental paper [21] and, again Section III.5.4 in [34]. Since its introduction in the thirties by Karl Dickman for the study of the asymptotic behavior of the frequency of numbers containing prime factors of a certain relative magnitude (see [9]), the Dickman's function has revealed its usefulness in a large variety of problems, not only of number-theoretic nature: we refer to the paper [23], where an extensive list of references is given. We only add the recent paper [7].

In particular, if we denote the Euler's constant by $\gamma$, we get the equality $\int_{0}^{\infty} \rho(x) \mathrm{d} x=\mathrm{e}^{\gamma}$ and the moment generating function (1.7) by Theorem 7 in Section III.5.4 in [34]. The moment generating function (1.7) can also be found in other references see e.g. equation (2.5) in [20] (where $K(\alpha)$ is identically equal to 1 ) and equation (66) in [1]. The probability density function $f(x):=\mathrm{e}^{-\gamma} \rho(x) 1_{(0, \infty)}(x)$ concerns a random variable $Z \stackrel{d}{\sim} \mathcal{D}(1)$

The outline of the paper is as follows. The weak convergence results for $\left\{W_{n}: n \geq 1\right\}$ in (1.4) (for some choices of the sequence $\left.\left\{R_{n}: n \geq 1\right\}\right)$ and the main large deviation results for the weighted means $\left\{Z_{n}: n \geq 1\right\}$ in (1.1) (for some choices of the sequence $\left\{W_{n}: n \geq 1\right\}$ which converges weakly to $\mathcal{D}(\nu)$ ) are proved in Sections 2 and 3 , respectively. In Section 4 we apply one of our weak convergence results and we recover a result of [5], where a probabilistic model for square-free numbers is introduced; this shows that our approach is useful because we do not need to consider specific properties of the prime numbers sequence. Finally, in Section 5, we discuss condition $(\mathbf{H})$ : we illustrate how the (common) rate function can be expressed in terms of the Hellinger's distance with respect to the weak limit $P_{W_{\infty}}=\mathcal{D}(\nu)$ of $\left\{W_{n}: n \geq 1\right\}$, and we present a counterexample in which condition condition $(\mathbf{H})$ does not hold. 


\section{WEAK CONVERGENCE RESUlts}

In this section we prove weak convergence results for the sequence $\left\{W_{n}: n \geq 1\right\}$ in (1.4) when the sequence $\left\{R_{n}: n \geq 1\right\}$ is as in the following examples.

Example 2.1. Let $\left\{\lambda_{n}: n \geq 1\right\}$ be a sequence of positive numbers such that (1.5) holds. Let $\left\{R_{n}: n \geq 1\right\}$ be independent random variables such that $R_{n} \stackrel{d}{\sim} \mathcal{P}\left(\lambda_{n}\right)$ for all $n \geq 1$.

Example 2.2. Let $\left\{R_{n}: n \geq 1\right\}$ be independent, nonnegative and integer valued random variables such that

$$
\lim _{n \rightarrow \infty} \frac{1}{\phi(n)} \sum_{k=1}^{n} \phi(k) \mathbb{E}\left[R_{k}\right]=\nu \text { and } \lim _{n \rightarrow \infty} \frac{1}{\phi(n)} \sum_{k=1}^{n} \phi(k) P\left(R_{k}=1\right)=\nu .
$$

We prove Propositions 2.5 and 2.6 which concern Examples 2.1 and 2.2, respectively. In this way we generalize Lemmas 1 and 2 in [1] (see Sect. 4.3). Proposition 2.6 allows to recover the weak convergence result in [5] as a particular case (see Sect. 4 for details). The following lemmas are useful.

Lemma 2.3. Let $\left\{a_{n}: n \geq 1\right\}$ and $\left\{b_{k}^{(n)}: n \geq k \geq 1\right\}$ be complex numbers such that

$$
\begin{gathered}
\lim _{n \rightarrow \infty} a_{n}=a \text { for some } a \in \mathbb{C}, \\
\lim _{n \rightarrow \infty} \sum_{k=1}^{n} b_{k}^{(n)}=b \text { for some } b \in \mathbb{C}, \\
\lim _{n \rightarrow \infty} b_{k}^{(n)}=0 \text { for all } k \geq 1, \\
C:=\sup \left\{\sum_{k=1}^{n}\left|b_{k}^{(n)}\right|: n \geq 1\right\}<\infty .
\end{gathered}
$$

Then: (i) $\lim _{n \rightarrow \infty} \sum_{k=1}^{n} a_{k} b_{k}^{(n)}=a b$; (ii) $\lim _{n \rightarrow \infty} \sum_{k=1}^{n-1} a_{k} b_{k}^{(n)}=a b$ if $\lim _{n \rightarrow \infty} b_{n}^{(n)}=0$.

Proof. Statement (i) is a part of Toeplitz's Lemma in [29] (p. 250). Moreover, since

$$
\sum_{k=1}^{n-1} a_{k} b_{k}^{(n)}=\sum_{k=1}^{n} a_{k} b_{k}^{(n)}-a_{n} b_{n}^{(n)}
$$

statement (ii) follows from (i), $\lim _{n \rightarrow \infty} b_{n}^{(n)}=0$ and (2.2).

Lemma 2.4. Assume that (1.2) and (1.5) hold. Then $\lim _{n \rightarrow \infty} \lambda_{n}=0$.

Proof. Just notice that

$$
\lambda_{n}=\frac{1}{\phi(n)} \sum_{k=1}^{n} \phi(k) \lambda_{k}-\frac{\phi(n-1)}{\phi(n)} \cdot \frac{1}{\phi(n-1)} \sum_{k=1}^{n-1} \phi(k) \lambda_{k} \rightarrow \nu-\nu=0 \text { as } n \rightarrow \infty .
$$

Now we are ready to prove the weak convergence results for each one of the examples above.

Proposition 2.5. Let $\phi: \mathbb{N} \rightarrow[0, \infty)$ be a strictly increasing function such that (1.2) holds. Let $\left\{\lambda_{n}: n \geq 1\right\}$ and $\left\{R_{n}: n \geq 1\right\}$ be as in Example 2.1. Then $\left\{W_{n}: n \geq 1\right\}$ in (1.4) converges weakly to $P_{W_{\infty}}=\mathcal{D}(\nu)$ as $n \rightarrow \infty$. 
Proof. We have to check that

$$
\lim _{n \rightarrow \infty} \mathbb{E}\left[\mathrm{e}^{s W_{n}}\right]=\exp \left(\nu \int_{0}^{1} \frac{\mathrm{e}^{s y}-1}{y} \mathrm{~d} y\right)(\text { for all } s \in \mathbb{R})
$$

by (1.7). The moment generating function of $W_{n}$ is

$$
\mathbb{E}\left[\mathrm{e}^{s W_{n}}\right]=\prod_{k=1}^{n} \exp \left(\lambda_{k}\left\{\mathrm{e}^{s \frac{\phi(k)}{\phi(n)}}-1\right\}\right)=\exp \left(\sum_{k=1}^{n} \lambda_{k}\left\{\mathrm{e}^{s \frac{\phi(k)}{\phi(n)}}-1\right\}\right) .
$$

By Abel's partial summation formula, we have

$$
\begin{aligned}
\sum_{k=1}^{n} \lambda_{k}\left\{\mathrm{e}^{s \frac{\phi(k)}{\phi(n)}}-1\right\} & =\sum_{k=1}^{n} \phi(k) \lambda_{k} \frac{\mathrm{e}^{s \frac{\phi(k)}{\phi(n)}}-1}{\phi(k)} \\
& =\sum_{k=1}^{n} \phi(k) \lambda_{k} \frac{\mathrm{e}^{s \frac{\phi(n)}{\phi(n)}}-1}{\phi(n)}-\sum_{k=1}^{n-1} \sum_{j=1}^{k} \phi(j) \lambda_{j}\left(\frac{\mathrm{e}^{s \frac{\phi(k+1)}{\phi(n)}}-1}{\phi(k+1)}-\frac{\mathrm{e}^{s \frac{\phi(k)}{\phi(n)}}-1}{\phi(k)}\right),
\end{aligned}
$$

and therefore

$$
\sum_{k=1}^{n} \lambda_{k}\left\{\mathrm{e}^{s \frac{\phi(k)}{\phi(n)}}-1\right\}=\frac{1}{\phi(n)} \sum_{k=1}^{n} \phi(k) \lambda_{k}\left(\mathrm{e}^{s}-1\right)-\sum_{k=1}^{n-1} a_{k} b_{k}^{(n)},
$$

where

$$
a_{k}:=\frac{1}{\phi(k)} \sum_{j=1}^{k} \phi(j) \lambda_{j} \text { and } b_{k}^{(n)}:=\phi(k)\left(\frac{\mathrm{e}^{s \frac{\phi(k+1)}{\phi(n)}}-1}{\phi(k+1)}-\frac{\mathrm{e}^{s \frac{\phi(k)}{\phi(n)}}-1}{\phi(k)}\right) .
$$

Now we take the limit as $n \rightarrow \infty$ in (2.6) and, by (1.5), we have to show that

$$
\lim _{n \rightarrow \infty} \sum_{k=1}^{n-1} a_{k} b_{k}^{(n)}=\nu b, \text { where } b:=\mathrm{e}^{s}-1-\int_{0}^{1} \frac{\mathrm{e}^{s x}-1}{x} \mathrm{~d} x .
$$

This will be done by applying Lemma 2.3 for the coefficients in (2.7). Firstly (2.2) holds with $a=\nu$ by (1.5); (2.4) and $b_{n}^{(n)} \rightarrow 0$ can be easily checked by inspection (we recall that $\phi(n) \rightarrow \infty$ and $\frac{\phi(n)}{\phi(n+1)} \rightarrow 1$ by (1.2)); if we consider (2.7) with $\lambda_{k}=\frac{\phi(k)-\phi(k-1)}{\phi(k)}$ for all $k \geq 1$ (as in Rem. 1.1), and therefore $a_{k}=1$ for all $k \geq 1$ (we recall that $\phi(0)=0)$, by $(2.6)$ we have

$$
\sum_{k=1}^{n} b_{k}^{(n)}=\mathrm{e}^{s}-1-\sum_{k=1}^{n} \frac{\phi(k)-\phi(k-1)}{\phi(k)}\left\{\mathrm{e}^{s \frac{\phi(k)}{\phi(n)}}-1\right\}+b_{n}^{(n)}
$$

and this yields (2.3) with $b$ as in (2.8) because

$$
\sum_{k=1}^{n} \frac{\phi(k)-\phi(k-1)}{\phi(k)}\left\{\mathrm{e}^{s \frac{\phi(k)}{\phi(n)}}-1\right\}=\sum_{k=1}^{n} \frac{\phi(k)-\phi(k-1)}{\phi(n)} \cdot \frac{\mathrm{e}^{s \frac{\phi(k)}{\phi(n)}}-1}{\phi(k) / \phi(n)}
$$

is a suitable Riemann sum for the integral $\int_{0}^{1} \frac{\mathrm{e}^{s x}-1}{x} \mathrm{~d} x ;(2.5)$ follows from (2.3) because $\left\{b_{k}^{(n)}: n-1 \geq k \geq 0\right\}$ are nonnegative numbers (since, for each fixed $s \in \mathbb{R}$, the function $x \mapsto \frac{\mathrm{e}^{s x}-1}{x}$ is nondecreasing, and the function $\phi$ is increasing).

Proposition 2.6. Let $\phi: \mathbb{N} \rightarrow[0, \infty)$ be a strictly increasing function such that (1.2) holds. Let $\left\{R_{n}: n \geq 1\right\}$ be as in Example 2.2. Then $\left\{W_{n}: n \geq 1\right\}$ in (1.4) converges weakly to $P_{W_{\infty}}=\mathcal{D}(\nu)$ as $n \rightarrow \infty$. 
Proof. We follow the same lines as the proof of Lemma 2 in [1], and the above Proposition 2.5 plays the role of Lemma 1 in [1]. The only difference is that here we have the coefficients $\{\phi(n): n \geq 1\}$ instead of $\phi(n)=n$ as in [1]. We set $\tilde{R}_{n}:=R_{n} \wedge 1$, and we have $\tilde{R}_{n} \leq R_{n}$ and $P\left(R_{n}=1\right) \leq \mathbb{E}\left[\tilde{R}_{n}\right] \leq \mathbb{E}\left[R_{n}\right]$ (for all $n \geq 1$ ). Moreover, if we set $\lambda_{n}:=\mathbb{E}\left[\tilde{R}_{n}\right]$, conditions (1.5) and

$$
\lim _{n \rightarrow \infty} \frac{1}{\phi(n)} \sum_{k=1}^{n} \phi(k)\left|\mathbb{E}\left[R_{k}\right]-\mathbb{E}\left[\tilde{R}_{k}\right]\right|=0
$$

hold by (2.1). So it remains to show that $\tilde{W}_{n}:=\frac{1}{\phi(n)} \sum_{k=1}^{n} \phi(k) \tilde{R}_{k}$ converges weakly to $\mathcal{D}(\nu)$ (as $\left.n \rightarrow \infty\right)$. In order to do that we can construct (as in Lem. 2 in [1]) a sequence of independent random variables $\left\{R_{n}^{*}: n \geq 1\right\}$ such that $R_{n}^{*} \stackrel{d}{\sim} \mathcal{P}\left(\lambda_{n}\right)$ for all $n \geq 1$ and

$$
0 \leq \frac{1}{\phi(n)} \sum_{k=1}^{n} \phi(k)\left|\mathbb{E}\left[\tilde{R}_{k}\right]-\mathbb{E}\left[R_{k}^{*}\right]\right| \leq \frac{1}{\phi(n)} \sum_{k=1}^{n} \phi(k) \lambda_{k}^{2}
$$

then, by Proposition 2.5, we only have to prove

$$
\lim _{n \rightarrow \infty} \frac{1}{\phi(n)} \sum_{k=1}^{n} \phi(k) \lambda_{k}^{2}=0 .
$$

To this extent we recall that $\lambda_{n} \rightarrow 0$ by Lemma 2.4 ; thus, for all $\varepsilon>0$, there exists $n_{0} \geq 1$ such that $\lambda_{n}<\varepsilon$ for all $n>n_{0}$; therefore, for $n>n_{0}$, we have

$$
0 \leq \sum_{k=1}^{n} \phi(k) \lambda_{k}^{2}=\sum_{k=1}^{n_{0}} \phi(k) \lambda_{k}^{2}+\varepsilon \sum_{k=n_{0}+1}^{n} \phi(k) \lambda_{k}=\sum_{k=1}^{n_{0}} \phi(k) \lambda_{k}^{2}+\varepsilon\left(\sum_{k=1}^{n} \phi(k) \lambda_{k}-\sum_{k=1}^{n_{0}} \phi(k) \lambda_{k}\right)
$$

and, if we divide by $\phi(n)$, we get (2.9) by (1.5) and by the arbitrariness of $\varepsilon>0$.

\section{LARGE DEVIATion RESUlts}

In this section we prove some LDPs for weighted means $\left\{Z_{n}: n \geq 1\right\}$ defined by (1.1), which are governed by the good rate function $I$ defined by (1.6). The following hypotheses on the strictly increasing function $\phi: \mathbb{N} \rightarrow[0, \infty)$ in (1.2) play a crucial role:

- the function $\phi$ is concave, i.e.

$$
\{\phi(n)-\phi(n-1): n \geq 1\} \text { is non-increasing; }
$$

- $\lim _{n \rightarrow \infty} L(n)=\infty$, where $L(n)$ is defined by (1.3).

Moreover we set

$$
s_{j, n}:=\sum_{k=j}^{n} \frac{\phi(k)-\phi(k-1)}{\phi^{2}(k)} .
$$

In the sequel the following lemma is useful.

Lemma 3.1. Let $\phi: \mathbb{N} \rightarrow[0, \infty)$ be the strictly increasing function in (1.2) and let $L(n)$ be defined by (1.3).

(i) Assume that (3.1) holds. Then $s_{1, \infty}<\infty$; for $n>j \geq 1$, we have

$$
\phi(j) s_{j, n}-\phi(j+1) s_{j+1, n} \geq 0
$$

for $n \geq j \geq 2$, we have

$$
1-\frac{\phi(j)}{\phi(n+1)} \leq \phi(j) s_{j, n} \leq \frac{\phi(j)}{\phi(j-1)} \leq c, \text { for some } c \geq \frac{\phi(n)}{\phi(n-1)} \text { for all } n \geq 2 .
$$


(ii) Assume that (1.5) and $\lim _{n \rightarrow \infty} L(n)=\infty$ hold. Then

$$
\lim _{n \rightarrow \infty} \frac{1}{L(n)} \sum_{k=1}^{n} \lambda_{k}=\nu
$$

Proof. Throughout this proof we call $\phi$ the piecewise linear function on $[0, \infty)$ which interpolates the values $\{\phi(n): n \geq 1\}$.

We start with the proof of part (i). We have

$$
\frac{1}{\phi(j)}-\frac{1}{\phi(n)}=\int_{j}^{n} \frac{\phi^{\prime}(x)}{\phi^{2}(x)} \mathrm{d} x=\sum_{k=j+1}^{n} \int_{k-1}^{k} \frac{\phi^{\prime}(x)}{\phi^{2}(x)} \mathrm{d} x \geq \sum_{k=j+1}^{n} \frac{1}{\phi^{2}(k)} \int_{k-1}^{k} \phi^{\prime}(x) \mathrm{d} x=s_{j+1, n},
$$

and we easily get $s_{1, \infty}<\infty$. Now the proof of (3.2). We have $\frac{1}{\phi(j)}-s_{j+1, n} \geq \frac{1}{\phi(n)} \geq 0$ by (3.5) and, since

$$
\begin{aligned}
\phi(j) s_{j, n}-\phi(j+1) s_{j+1, n} & =\phi(j)\left\{\frac{\phi(j)-\phi(j-1)}{\phi^{2}(j)}+s_{j+1, n}\right\}-\phi(j+1) s_{j+1, n} \\
& =\frac{\phi(j)-\phi(j-1)}{\phi(j)}-(\phi(j+1)-\phi(j)) s_{j+1, n}
\end{aligned}
$$

by the concavity condition (3.1) (together with $\frac{1}{\phi(j)}-s_{j+1, n} \geq 0$ obtained above) we have

$$
\phi(j) s_{j, n}-\phi(j+1) s_{j+1, n} \geq(\phi(j+1)-\phi(j))\left\{\frac{1}{\phi(j)}-s_{j+1, n}\right\} \geq 0 .
$$

We conclude with the proof of (3.3). We have

$$
s_{j, n} \geq \sum_{k=j}^{n} \frac{\phi(k+1)-\phi(k)}{\phi^{2}(k)}=\sum_{k=j}^{n} \frac{1}{\phi^{2}(k)} \int_{k}^{k+1} \phi^{\prime}(x) \mathrm{d} x \geq \sum_{k=j}^{n} \int_{k}^{k+1} \frac{\phi^{\prime}(x)}{\phi^{2}(x)} \mathrm{d} x=-\frac{1}{\phi(n+1)}+\frac{1}{\phi(j)}
$$

(the first inequality holds by (3.1)) and $s_{j, n} \leq \frac{1}{\phi(j-1)}$ by (3.5); thus we get (3.3) by multiplying by $\phi(j)$, and the existence of a finite value $c$ such that $c \geq \frac{\phi(n)}{\phi(n-1)}$ for all $n \geq 2$ follows from $\lim _{n \rightarrow \infty} \frac{\phi(n)}{\phi(n+1)}=1$ (see (1.2)). Now we prove part (ii). By Abel's partial summation formula and some computations, we can write

$$
\sum_{k=1}^{n} \lambda_{k}=\sum_{k=1}^{n}\left(\phi(k) \lambda_{k}\right) \frac{1}{\phi(k)}=\frac{1}{\phi(n)} \sum_{k=1}^{n} \phi(k) \lambda_{k}+\sum_{k=1}^{n-1}\left(\frac{1}{\phi(k)} \sum_{j=1}^{k} \phi(j) \lambda_{j}\right) \frac{\phi(k+1)-\phi(k)}{\phi(k+1)} .
$$

Hence

$$
\frac{1}{L(n)} \sum_{k=1}^{n} \lambda_{k}=\frac{1}{\phi(n) L(n)} \sum_{k=1}^{n} \phi(k) \lambda_{k}+\sum_{k=1}^{n-1} a_{k} b_{k}^{(n)}
$$

where

$$
a_{k}:=\frac{1}{\phi(k)} \sum_{j=1}^{k} \phi(j) \lambda_{j} \text { and } b_{k}^{(n)}:=\frac{\phi(k+1)-\phi(k)}{\phi(k+1) L(n)} .
$$

Note that $\frac{1}{\phi(n) L(n)} \sum_{k=1}^{n} \phi(k) \lambda_{k} \rightarrow 0$ by (1.5) and $\lim _{n \rightarrow \infty} L(n)=\infty$; therefore we complete the proof if we show that

$$
\lim _{n \rightarrow \infty} \sum_{k=1}^{n-1} a_{k} b_{k}^{(n)}=\nu .
$$


This will be done by applying Lemma 2.3. Firstly (2.2) holds with $a=\nu$ by $(1.5) ;(2.4)$ and $b_{n}^{(n)} \rightarrow 0$ can be easily checked by inspection (we recall that $L(n) \rightarrow \infty$ and, by (1.2), $\frac{\phi(n)}{\phi(n+1)} \rightarrow 1$ ); (2.3) holds with $b=1$ noting that

$$
\sum_{k=1}^{n} b_{k}^{(n)}=\frac{1}{L(n)} \sum_{k=1}^{n-1} \frac{\phi(k+1)-\phi(k)}{\phi(k+1)}+b_{n}^{(n)}=\frac{L(n)-1}{L(n)}+b_{n}^{(n)} \rightarrow 1 \text { as } n \rightarrow \infty ;
$$

(2.5) follows from (2.3) because $\left\{b_{k}^{(n)}: n-1 \geq k \geq 0\right\}$ are nonnegative numbers (we recall that the function $\phi$ is increasing).

Now we are ready to prove the first LDP.

Proposition 3.2. Let $\phi: \mathbb{N} \rightarrow[0, \infty)$ be a strictly increasing function such that (1.2), (3.1) and $\lim _{n \rightarrow \infty} L(n)=\infty$ hold. Let $\left\{\lambda_{n}: n \geq 1\right\}$ and $\left\{R_{n}: n \geq 1\right\}$ be as in Example 2.1. Then $\left\{Z_{n}: n \geq 1\right\}$ defined by (1.1) and (1.4) satisfies the LDP with speed function $v_{n}=L(n)$ and good rate function $I$ defined by (1.6).

Proof. We prove that

$$
\lim _{n \rightarrow \infty} \frac{1}{L(n)} \log \mathbb{E}\left[\mathrm{e}^{\theta \sum_{k=1}^{n} \frac{\phi(k)-\phi(k-1)}{\phi^{2}(k)} \sum_{j=1}^{k} \phi(j) R_{j}}\right]=\nu\left(\mathrm{e}^{\theta}-1\right)
$$

for all $\theta \in \mathbb{R}$. Since the right hand side above is finite and differentiable for all $\theta \in \mathbb{R}$, the LDP holds by the Gärtner Ellis Theorem with good rate function $I$ defined by the Legendre transform

$$
I(x):=\sup _{\theta \in \mathbb{R}}\left\{\theta x-\nu\left(\mathrm{e}^{\theta}-1\right)\right\},
$$

which coincides with the rate function $I$ in the statement of the proposition. It is useful to handle the above expression as follows. Since the random variables $\left\{R_{n}: n \geq 1\right\}$ are independent and $R_{n} \stackrel{d}{\sim} \mathcal{P}\left(\lambda_{n}\right)$ for all $n \geq 1$, we have

$$
\begin{aligned}
\log \mathbb{E}\left[\mathrm{e}^{\theta \sum_{k=1}^{n} \frac{\phi(k)-\phi(k-1)}{\phi^{2}(k)} \sum_{j=1}^{k} \phi(j) R_{j}}\right] & =\log \mathbb{E}\left[\mathrm{e}^{\theta \sum_{j=1}^{n} \phi(j) R_{j} s_{j, n}}\right] \\
& =\sum_{j=1}^{n} \log \mathbb{E}\left[\mathrm{e}^{\theta \phi(j) R_{j} s_{j, n}}\right]=\sum_{j=1}^{n} \lambda_{j}\left(\mathrm{e}^{\theta \phi(j) s_{j, n}}-1\right) ;
\end{aligned}
$$

thus we have to prove that

$$
\lim _{n \rightarrow \infty} \frac{1}{L(n)} \sum_{j=1}^{n} \lambda_{j}\left(\mathrm{e}^{\theta \phi(j) s_{j, n}}-1\right)=\nu\left(\mathrm{e}^{\theta}-1\right)
$$

for all $\theta \in \mathbb{R}$.

The case $\theta=0$ is immediate. We give all the details only for the case $\theta>0$ because the case $\theta<0$ can be treated similarly. Firstly, by taking into account $s_{1, \infty}<\infty$ and (3.3) with the constant $c$ therein (see Lem. 3.1) and $\frac{\phi(n)}{\phi(n-1)} \rightarrow 1$ (see (1.2)), for all $\varepsilon>0$ there exists $j_{0} \geq 2$ such that for $n \geq j>j_{0}$ we have $\phi(j) s_{j, n} \leq 1+\varepsilon$; therefore

$$
\sum_{j=1}^{n} \lambda_{j}\left(\mathrm{e}^{\theta \phi(j) s_{j, n}}-1\right) \leq \lambda_{1}\left(\mathrm{e}^{\theta \phi(1) s_{1, \infty}}-1\right)+\sum_{j=2}^{j_{0}} \lambda_{j}\left(\mathrm{e}^{\theta c}-1\right)+\sum_{j=j_{0}+1}^{n} \lambda_{j}\left(\mathrm{e}^{\theta(1+\varepsilon)}-1\right)
$$

and, if we divide by $L(n)$, we get

$$
\limsup _{n \rightarrow \infty} \frac{1}{L(n)} \sum_{j=1}^{n} \lambda_{j}\left(\mathrm{e}^{\theta \phi(j) s_{j, n}}-1\right) \leq \nu\left(\mathrm{e}^{\theta}-1\right)
$$


by (3.4) and the arbitrariness of $\varepsilon$. Now we prove the matching lower bound. We start noting that, by Abel's partial summation formula, we have

$$
\begin{aligned}
\sum_{j=1}^{n} \lambda_{j}\left(\mathrm{e}^{\theta \phi(j) s_{j, n}}-1\right) & =\sum_{j=1}^{n} \lambda_{j}\left(\mathrm{e}^{\theta \frac{\phi(n)-\phi(n-1)}{\phi(n)}}-1\right)+\sum_{j=1}^{n-1} \sum_{k=1}^{j} \lambda_{k}\left(\mathrm{e}^{\theta \phi(j) s_{j, n}}-\mathrm{e}^{\theta \phi(j+1) s_{j+1, n}}\right) \\
& \geq \sum_{j=1}^{n-1} \sum_{k=1}^{j} \lambda_{k}\left(\mathrm{e}^{\theta \phi(j) s_{j, n}}-\mathrm{e}^{\theta \phi(j+1) s_{j+1, n}}\right) .
\end{aligned}
$$

Moreover $\mathrm{e}^{\theta \phi(j) s_{j, n}}-\mathrm{e}^{\theta \phi(j+1) s_{j+1, n}} \geq 0$ by (3.2); then, by (3.4), for all $\varepsilon \in(0, \nu)$ there exists $n_{0}$ such that for $n>n_{0}+1$ we have

$$
\begin{aligned}
\sum_{j=1}^{n-1} \sum_{k=1}^{j} \lambda_{k}\left(\mathrm{e}^{\theta \phi(j) s_{j, n}}-\mathrm{e}^{\theta \phi(j+1) s_{j+1, n}}\right) \geq & \sum_{j=1}^{n_{0}} \sum_{k=1}^{j} \lambda_{k}\left(\mathrm{e}^{\theta \phi(j) s_{j, n}}-\mathrm{e}^{\theta \phi(j+1) s_{j+1, n}}\right) \\
& +(\nu-\varepsilon) \sum_{j=n_{0}+1}^{n-1} L(j)\left(\mathrm{e}^{\theta \phi(j) s_{j, n}}-\mathrm{e}^{\theta \phi(j+1) s_{j+1, n}}\right)
\end{aligned}
$$

and (as far as the last sum is concerned)

$$
\begin{aligned}
& \sum_{j=n_{0}+1}^{n-1} L(j)\left(\mathrm{e}^{\theta \phi(j) s_{j, n}}-\mathrm{e}^{\theta \phi(j+1) s_{j+1, n}}\right) \\
& \quad=\sum_{j=1}^{n-1} L(j)\left(\mathrm{e}^{\theta \phi(j) s_{j, n}}-\mathrm{e}^{\theta \phi(j+1) s_{j+1, n}}\right)-\sum_{j=1}^{n_{0}} L(j)\left(\mathrm{e}^{\theta \phi(j) s_{j, n}}-\mathrm{e}^{\theta \phi(j+1) s_{j+1, n}}\right) ;
\end{aligned}
$$

thus, by putting the pieces together (we recall that $0 \leq \mathrm{e}^{\theta \phi(j) s_{j, n}}-\mathrm{e}^{\theta \phi(j+1) s_{j+1, n}} \leq \mathrm{e}^{\theta \phi(j) s_{1, \infty}}$ with $s_{1, \infty}<\infty$, and the finite sums are negligible when we divide by $L(n)$ because $L(n) \rightarrow \infty$ ), we obtain

$$
\liminf _{n \rightarrow \infty} \frac{1}{L(n)} \sum_{j=1}^{n} \lambda_{j}\left(\mathrm{e}^{\theta \phi(j) s_{j, n}}-1\right) \geq(\nu-\varepsilon) \liminf _{n \rightarrow \infty} \frac{1}{L(n)} \sum_{j=1}^{n-1} L(j)\left(\mathrm{e}^{\theta \phi(j) s_{j, n}}-\mathrm{e}^{\theta(j+1) s_{j+1, n}}\right) .
$$

Now we concentrate our attention on the last right hand side here above. From Abel's partial summation formula we get

$$
\begin{aligned}
& \sum_{j=1}^{n-1} L(j)\left(\mathrm{e}^{\theta \phi(j) s_{j, n}}-\mathrm{e}^{\theta \phi(j+1) s_{j+1, n}}\right) \\
& =\sum_{j=1}^{n} \frac{\phi(j)-\phi(j-1)}{\phi(j)}\left(\mathrm{e}^{\theta \phi(j) s_{j, n}}-1\right)-\sum_{j=1}^{n} \frac{\phi(j)-\phi(j-1)}{\phi(j)}\left(\mathrm{e}^{\theta \phi(n) s_{n, n}}-1\right) \\
& =\mathrm{e}^{\theta \phi(1) s_{1, n}}-1+\sum_{j=2}^{n} \frac{\phi(j)-\phi(j-1)}{\phi(j)}\left(\mathrm{e}^{\theta \phi(j) s_{j, n}}-1\right)-L(n)\left(\mathrm{e}^{\theta\left(1-\frac{\phi(n-1)}{\phi(n)}\right)}-1\right) ;
\end{aligned}
$$

then, by (3.3) and $\phi(1) s_{1, n}>0$, we have

$$
\sum_{j=1}^{n-1} L(j)\left(\mathrm{e}^{\theta \phi(j) s_{j, n}}-\mathrm{e}^{\theta \phi(j+1) s_{j+1, n}}\right) \geq \sum_{j=2}^{n} \frac{\phi(j)-\phi(j-1)}{\phi(j)}\left(\mathrm{e}^{\theta\left(1-\frac{\phi(j)}{\phi(n+1)}\right)}-1\right)-L(n)\left(\mathrm{e}^{\theta\left(1-\frac{\phi(n-1)}{\phi(n)}\right)}-1\right),
$$


and therefore we obtain

$$
\liminf _{n \rightarrow \infty} \frac{1}{L(n)} \sum_{j=1}^{n} \lambda_{j}\left(\mathrm{e}^{\theta \phi(j) s_{j, n}}-1\right) \geq(\nu-\varepsilon) \liminf _{n \rightarrow \infty} \frac{1}{L(n)} \sum_{j=2}^{n} \frac{\phi(j)-\phi(j-1)}{\phi(j)}\left(\mathrm{e}^{\theta\left(1-\frac{\phi(j)}{\phi(n+1)}\right)}-1\right)
$$

by taking into account that $\frac{\phi(n)}{\phi(n+1)} \rightarrow 1$ (see (1.2)). Finally we get

$$
\begin{aligned}
\liminf _{n \rightarrow \infty} \frac{1}{L(n)} \sum_{j=1}^{n} \lambda_{j}\left(\mathrm{e}^{\theta \phi(j) s_{j, n}}-1\right) \geq & (\nu-\varepsilon) \liminf _{n \rightarrow \infty} \frac{1}{L(n)} \sum_{j=2}^{n} \frac{\phi(j)-\phi(j-1)}{\phi(j)}\left(\mathrm{e}^{\theta\left(1-\frac{\phi(j)}{\phi(n+1)}\right)}-1\right) \\
\geq & (\nu-\varepsilon)\left(\liminf _{n \rightarrow \infty} \frac{\mathrm{e}^{\theta}}{L(n)} \sum_{j=2}^{n} \frac{\phi(j)-\phi(j-1)}{\phi(j)}\left(\mathrm{e}^{-\theta \frac{\phi(j)}{\phi(n+1)}}-1\right)\right. \\
& \left.+\liminf _{n \rightarrow \infty} \frac{1}{L(n)} \sum_{j=2}^{n} \frac{\phi(j)-\phi(j-1)}{\phi(j)}\left(\mathrm{e}^{\theta}-1\right)\right),
\end{aligned}
$$

with some manipulations, and therefore

$$
\liminf _{n \rightarrow \infty} \frac{1}{L(n)} \sum_{j=1}^{n} \lambda_{j}\left(\mathrm{e}^{\theta \phi(j) s_{j, n}}-1\right) \geq \nu\left(\mathrm{e}^{\theta}-1\right)
$$

by taking into account that

$$
0 \geq \liminf _{n \rightarrow \infty} \sum_{j=2}^{n} \frac{\phi(j)-\phi(j-1)}{\phi(j)}\left(\mathrm{e}^{-\theta \frac{\phi(j)}{\phi(n+1)}}-1\right)=\int_{0}^{1} \frac{\mathrm{e}^{-\theta x}-1}{x} \mathrm{~d} x>-\infty
$$

(we are dealing with a Riemann sum for the indicated integral) and by the arbitrariness of $\varepsilon$.

In what follows a symbol $*$ will be attached to all items in Proposition 3.2. Thus we have $\phi^{*}, L^{*}(n)$, $\left\{s_{j, n}^{*}: n \geq j \geq 1\right\},\left\{\lambda_{n}^{*}: n \geq 1\right\}$ and $\left\{R_{n}^{*}: n \geq 1\right\}$, and all the hypotheses on them. Our aim is to prove a result without the concavity assumption (3.1); however, in some sense, $\phi$ needs to exhibit the same behavior as the concave function in Proposition 3.2, i.e. we require that

$$
\lim _{n \rightarrow \infty} \frac{\phi(n)-\phi(n-1)}{\phi^{*}(n)-\phi^{*}(n-1)}=1 .
$$

The following lemma will be useful and its proof is immediate.

Lemma 3.3. Let $\phi^{*}, L^{*}(n),\left\{s_{j, n}^{*}: n \geq j \geq 1\right\}$ and $\left\{\lambda_{n}^{*}: n \geq 1\right\}$ be the items in Proposition 3.2, with all the hypotheses on them. Let $\phi: \mathbb{N} \rightarrow[0, \infty)$ be a strictly increasing function and let $\left\{\lambda_{n}: n \geq 1\right\}$ be a sequence of positive numbers such that (1.2), (1.5) and (3.6) hold. Then:

$$
\begin{gathered}
\lim _{n \rightarrow \infty} \frac{\phi(n)-\phi(n-1)}{\phi^{*}(n+1)-\phi^{*}(n)}=1 ; \\
\lim _{n \rightarrow \infty} \frac{\phi(n)}{\phi^{*}(n)}=1 ; \\
L(n) \sim L^{*}(n) ; \\
\sum_{k=1}^{n} \lambda_{k} \sim \sum_{k=1}^{n} \lambda_{k}^{*} ; \\
s_{1, \infty}<\infty .
\end{gathered}
$$

Now we are ready to prove another LDP. 
Proposition 3.4. Let $\phi^{*}, L^{*}(n),\left\{s_{j, n}^{*}: n \geq j \geq 1\right\},\left\{\lambda_{n}^{*}: n \geq 1\right\}$ and $\left\{R_{n}^{*}: n \geq 1\right\}$ be the items in Proposition 3.2, with all the hypotheses on them. Let $\phi: \mathbb{N} \rightarrow[0, \infty)$ be a strictly increasing function such that (1.2) and (3.6) hold. Let $\left\{\lambda_{n}: n \geq 1\right\}$ and $\left\{R_{n}: n \geq 1\right\}$ be as in Example 2.1. Then $\left\{Z_{n}: n \geq 1\right\}$ defined by (1.1) and (1.4) satisfies the LDP with speed function $v_{n}=L(n)$ and good rate function I defined by (1.6).

Proof. We follow the same lines as Proposition 3.2 where we proved that

$$
\lim _{n \rightarrow \infty} \frac{1}{L^{*}(n)} \sum_{j=1}^{n} \lambda_{j}^{*}\left(\mathrm{e}^{\theta \phi^{*}(j) s_{j, n}^{*}}-1\right)=\nu\left(\mathrm{e}^{\theta}-1\right)
$$

for all $\theta \in \mathbb{R}$. Then, by also taking into account (3.9), we have to prove that

$$
\lim _{n \rightarrow \infty} \frac{1}{L^{*}(n)} \sum_{j=1}^{n} \lambda_{j}\left(\mathrm{e}^{\theta \phi(j) s_{j, n}}-1\right)=\nu\left(\mathrm{e}^{\theta}-1\right)
$$

for all $\theta \in \mathbb{R}$.

The case $\theta=0$ is immediate. We give all the details only for the case $\theta>0$ because the case $\theta<0$ can be treated similarly. Let $\varepsilon \in(0,1)$ be arbitrarily fixed; there exists $n_{0}$ such that for $n \geq j>n_{0}$ we have

$$
\begin{gathered}
(1-\varepsilon) \phi^{*}(n)<\phi(n)<(1+\varepsilon) \phi^{*}(n), \\
(1-\varepsilon) \sum_{k=1}^{n} \lambda_{k}^{*}<\sum_{k=1}^{n} \lambda_{k}<(1+\varepsilon) \sum_{k=1}^{n} \lambda_{k}^{*} \\
(1-\varepsilon) s_{j, n}^{*}<s_{j, n}<(1+\varepsilon) s_{j, n}^{*}
\end{gathered}
$$

by (3.8), (3.10) and (3.9) (together with Cesaro's Theorem and some computations), respectively. We give all the details only for the upper bound

$$
\limsup _{n \rightarrow \infty} \frac{1}{L^{*}(n)} \sum_{j=1}^{n} \lambda_{j}\left(\mathrm{e}^{\theta \phi(j) s_{j, n}}-1\right) \leq \nu\left(\mathrm{e}^{\theta}-1\right)
$$

because the matching lower bound can be proved similarly. Let $j_{0}$ be such that $n>j_{0}>n_{0}$ and write

$$
\sum_{j=1}^{n} \lambda_{j}\left(\mathrm{e}^{\theta \phi(j) s_{j, n}}-1\right)=\sum_{j=1}^{j_{0}} \lambda_{j}\left(\mathrm{e}^{\theta \phi(j) s_{j, n}}-1\right)+\sum_{j=j_{0}+1}^{n} \lambda_{j}\left(\mathrm{e}^{\theta \phi(j) s_{j, n}}-1\right) ;
$$

we have $0 \leq \frac{1}{L^{*}(n)} \sum_{j=1}^{j_{0}} \lambda_{j}\left(\mathrm{e}^{\theta \phi(j) s_{j, n}}-1\right) \leq \frac{1}{L^{*}(n)} \sum_{j=1}^{j_{0}} \lambda_{j}\left(\mathrm{e}^{\theta \phi(j) s_{1, \infty}}-1\right) \rightarrow 0$ because $s_{1, \infty}<\infty$; so we can take the second sum only into account, and we notice that

$$
\sum_{j=j_{0}+1}^{n} \lambda_{j}\left(\mathrm{e}^{\theta \phi(j) s_{j, n}}-1\right) \leq \sum_{j=j_{0}+1}^{n} \lambda_{j}\left(\mathrm{e}^{\theta(1+\varepsilon)^{2} \phi^{*}(j) s_{j, n}^{*}}-1\right)
$$

by (3.11) and (3.13). Moreover, by a standard argument, we can consider $\sum_{j=1}^{n} \lambda_{j}\left(\mathrm{e}^{\theta(1+\varepsilon)^{2} \phi^{*}(j) s_{j, n}^{*}}-1\right)$ in place of the last sum and, by Abel's partial summation formula, we get

$$
\begin{aligned}
& \sum_{j=1}^{n} \lambda_{j}\left(\mathrm{e}^{\theta(1+\varepsilon)^{2} \phi^{*}(j) s_{j, n}^{*}}-1\right) \\
& \quad=\sum_{j=1}^{n} \lambda_{j}\left(\mathrm{e}^{\theta(1+\varepsilon)^{2}\left(\frac{\phi^{*}(n)-\phi^{*}(n-1)}{\phi^{*}(n)}\right)}-1\right)+\sum_{k=1}^{n-1} \sum_{j=1}^{k} \lambda_{j}\left(\mathrm{e}^{\theta(1+\varepsilon)^{2} \phi^{*}(k) s_{k, n}^{*}}-\mathrm{e}^{\theta(1+\varepsilon)^{2} \phi^{*}(k+1) s_{k+1, n}^{*}}\right) .
\end{aligned}
$$


The first term can be neglected when we divide by $L^{*}(n)$ (and $n$ goes to infinity); in fact we have $0 \leq \frac{\phi^{*}(n)-\phi^{*}(n-1)}{\phi^{*}(n)} \leq \frac{\phi^{*}(1)}{\phi^{*}(n)} \rightarrow 0$ by (3.1) and $\phi^{*}(n) \rightarrow \infty$ (by (1.2)), and $\frac{1}{L^{*}(n)} \sum_{k=1}^{n} \lambda_{k} \rightarrow \nu$ (this is a consequence of (3.4) and (3.9)). So, by also taking into account (3.12), we estimate the second term as follows:

$$
\begin{aligned}
\sum_{k=1}^{n-1} \sum_{j=1}^{k} \lambda_{j} & \left(\mathrm{e}^{\theta(1+\varepsilon)^{2} \phi^{*}(k) s_{k, n}^{*}}-\mathrm{e}^{\theta(1+\varepsilon)^{2} \phi^{*}(k+1) s_{k+1, n}^{*}}\right) \\
= & \sum_{k=1}^{j_{0}} \sum_{j=1}^{k} \lambda_{j}\left(\mathrm{e}^{\theta(1+\varepsilon)^{2} \phi^{*}(k) s_{k, n}^{*}}-\mathrm{e}^{\theta(1+\varepsilon)^{2} \phi^{*}(k+1) s_{k+1, n}^{*}}\right) \\
& +\sum_{k=j_{0}+1}^{n-1} \sum_{j=1}^{k} \lambda_{j}\left(\mathrm{e}^{\theta(1+\varepsilon)^{2} \phi^{*}(k) s_{k, n}^{*}}-\mathrm{e}^{\theta(1+\varepsilon)^{2} \phi^{*}(k+1) s_{k+1, n}^{*}}\right) \\
\leq & \sum_{k=1}^{j_{0}} \sum_{j=1}^{k} \lambda_{j}\left(\mathrm{e}^{\theta(1+\varepsilon)^{2} \phi^{*}(k) s_{1, \infty}^{*}}\right) \\
& +(1+\varepsilon) \sum_{k=j_{0}+1}^{n-1} \sum_{j=1}^{k} \lambda_{j}^{*}\left(\mathrm{e}^{\theta(1+\varepsilon)^{2} \phi^{*}(k) s_{k, n}^{*}}-\mathrm{e}^{\theta(1+\varepsilon)^{2} \phi^{*}(k+1) s_{k+1, n}^{*}}\right) .
\end{aligned}
$$

The first term is finite and therefore can be neglected when we divide by $L^{*}(n)$ (and $n$ goes to infinity). So, by a standard argument (already used above), and with a slight change of the last term, by Abel's partial summation formula we get

$$
\begin{aligned}
\sum_{k=1}^{n-1} \sum_{j=1}^{k} \lambda_{j}^{*}\left(\mathrm{e}^{\theta(1+\varepsilon)^{2} \phi^{*}(k) s_{k, n}^{*}}-\mathrm{e}^{\theta(1+\varepsilon)^{2} \phi^{*}(k+1) s_{k+1, n}^{*}}\right) & \\
= & \sum_{j=1}^{n} \lambda_{j}^{*}\left(\mathrm{e}^{\theta(1+\varepsilon)^{2} \phi^{*}(j) s_{j, n}^{*}}-1\right)-\sum_{j=1}^{n} \lambda_{j}^{*}\left(\mathrm{e}^{\theta(1+\varepsilon)^{2} \phi^{*}(n) s_{n, n}^{*}}-1\right) .
\end{aligned}
$$

When we divide by $L^{*}(n)$ (and $n$ goes to infinity), we can use the content of the proof Proposition 3.2 for the first sum and we can neglect the second sum. In fact, for the second sum, we have $0 \leq \phi^{*}(n) s_{n, n}^{*} \leq \frac{\phi^{*}(1)}{\phi^{*}(n)}$ (and we know that $\frac{\phi^{*}(1)}{\phi^{*}(n)} \rightarrow 0$ ) and $\frac{1}{L^{*}(n)} \sum_{k=1}^{n} \lambda_{k}^{*} \rightarrow \nu$ (this is a consequence of $\frac{1}{L^{*}(n)} \sum_{k=1}^{n} \lambda_{k} \rightarrow \nu$ obtained above and (3.10)). In conclusion, by putting the pieces together, we have

$$
\limsup _{n \rightarrow \infty} \frac{1}{L^{*}(n)} \sum_{j=1}^{n} \lambda_{j}\left(\mathrm{e}^{\theta \phi(j) s_{j, n}}-1\right) \leq(1+\varepsilon) \nu\left(\mathrm{e}^{\theta(1+\varepsilon)^{2}}-1\right)
$$

and we conclude by letting $\varepsilon$ go to zero.

The next proposition provides another LDP for $\left\{Z_{n}: n \geq 1\right\}$ in (1.1), where $\left\{W_{n}: n \geq 1\right\}$ are defined by (1.4) and the random variables $\left\{R_{n}: n \geq 1\right\}$ are independent and $R_{n} \stackrel{d}{\sim} \mathcal{B}\left(1-\mathrm{e}^{-\lambda_{n}}\right)$ for all $n \geq 1$. The main tool in the proof is a standard large deviation technique (see e.g. Thm. 4.2.13 in [8]) based on the concept of exponential equivalence (see e.g. Def. 4.2 .10 in [8]).

Proposition 3.5. Let $\phi$ and $\left\{\lambda_{n}: n \geq 1\right\}$ be as in Proposition 3.2 or in Proposition 3.4. Let $\left\{W_{n}: n \geq 1\right\}$ be defined by (1.4) and let $\left\{R_{n}: n \geq 1\right\}$ be independent random variables such that $R_{n} \stackrel{d}{\sim} \mathcal{B}\left(1-\mathrm{e}^{-\lambda_{n}}\right)$ for all $n \geq 1$. Then $\left\{Z_{n}: n \geq 1\right\}$ in (1.1) satisfies the LDP with speed function $v_{n}=L(n)$ and good rate function $I$ defined by (1.6). 
Remark 3.6. Proposition 3.5 holds also if $R_{n} \stackrel{d}{\sim} \mathcal{B}\left(\lambda_{n}\right)$ (in place of $R_{n} \stackrel{d}{\sim} \mathcal{B}\left(1-\mathrm{e}^{-\lambda_{n}}\right)$ ), for $n$ large enough to have $\lambda_{n} \in(0,1)$. In fact, if we apply Proposition 3.5 to $\left\{\hat{\lambda}_{n}: n \geq 1\right\}$ defined by $\hat{\lambda}_{n}:=\log \frac{1}{1-\lambda_{n}}$, the sequence $\left\{1-\mathrm{e}^{-\hat{\lambda}_{n}}: n \geq 1\right\}$ coincides with $\left\{\lambda_{n}: n \geq 1\right\}$.

Proof of Proposition 3.5. In this proof $\left\{W_{n}^{*}: n \geq 1\right\}$ and $\left\{R_{n}^{*}: n \geq 1\right\}$ are the sequences defined as $\left\{W_{n}: n \geq 1\right\}$ and $\left\{R_{n}: n \geq 1\right\}$ in Proposition 3.2 or in Proposition 3.4; then, if we set $R_{n}:=R_{n}^{*} \wedge 1$ (for all $n \geq 1$ ), the sequence $\left\{R_{n}: n \geq 1\right\}$ is indeed a version of the sequence appearing in the statement of this proposition. Hence we prove the desired LDP by checking the exponential equivalence condition

$$
\limsup _{n \rightarrow \infty} \frac{1}{L(n)} \log P\left(\Delta_{n}>\delta\right)=-\infty(\text { for all } \delta>0),
$$

where

$$
\Delta_{n}:=\left|\frac{1}{L(n)} \sum_{k=1}^{n} \frac{\phi(k)-\phi(k-1)}{\phi(k)} W_{k}-\frac{1}{L(n)} \sum_{k=1}^{n} \frac{\phi(k)-\phi(k-1)}{\phi(k)} W_{k}^{*}\right| .
$$

Firstly

$$
\begin{aligned}
\Delta_{n} & =\left|\frac{1}{L(n)} \sum_{k=1}^{n} \frac{\phi(k)-\phi(k-1)}{\phi^{2}(k)} \sum_{j=1}^{k} \phi(j)\left(R_{j}-R_{j}^{*}\right)\right| \\
& =\frac{1}{L(n)}\left|\sum_{j=1}^{n} \phi(j) s_{j, n}\left(R_{j}-R_{j}^{*}\right)\right| \leq \frac{c \vee\left(\phi(1) s_{1, \infty}\right)}{L(n)} \sum_{j=1}^{n}\left|R_{j}-R_{j}^{*}\right|,
\end{aligned}
$$

where $c$ is as in (3.3). From now on in this proof we simply write $c$ in place of $c \vee\left(\phi(1) s_{1, \infty}\right)$. Hence

$$
P\left(\Delta_{n}>\delta\right) \leq P\left(\frac{c}{L(n)} \sum_{j=1}^{n}\left|R_{j}-R_{j}^{*}\right|>\delta\right)=P\left(\sum_{j=1}^{n}\left|R_{j}-R_{j}^{*}\right|>\frac{\delta}{c} L(n)\right) .
$$

For each arbitrarily fixed $\theta \geq 0$, we have

$$
P\left(\sum_{j=1}^{n}\left|R_{j}-R_{j}^{*}\right|>\frac{\delta}{c} L(n)\right) \leq \frac{\mathbb{E}\left[\mathrm{e}^{\theta \sum_{j=1}^{n}\left|R_{j}-R_{j}^{*}\right|}\right]}{\mathrm{e}^{\theta \frac{\delta}{c} L(n)}}=\frac{\prod_{j=1}^{n} \mathbb{E}\left[\mathrm{e}^{\theta\left|R_{j}-R_{j}^{*}\right|}\right]}{\mathrm{e}^{\theta \frac{\delta}{c} L(n)}}
$$

by the Chernoff bound, and therefore

$$
\frac{1}{L(n)} \log P\left(\Delta_{n}>\delta\right) \leq \frac{1}{L(n)} \sum_{j=1}^{n} \log \mathbb{E}\left[\mathrm{e}^{\theta\left|R_{j}-R_{j}^{*}\right|}\right]-\theta \frac{\delta}{c} .
$$

Moreover

$$
\begin{aligned}
\mathbb{E}\left[\mathrm{e}^{\theta\left|R_{j}-R_{j}^{*}\right|}\right] & =P\left(R_{j}^{*}=0\right)+P\left(R_{j}^{*}=1\right)+\sum_{h=2}^{\infty} \mathrm{e}^{\theta|1-h|} P\left(R_{j}^{*}=h\right) \\
& =\mathrm{e}^{-\lambda_{j}}+\lambda_{j} \mathrm{e}^{-\lambda_{j}}+\sum_{h=2}^{\infty} \mathrm{e}^{\theta(h-1)} \frac{\lambda_{j}^{h}}{h !} \mathrm{e}^{-\lambda_{j}} \\
& =\mathrm{e}^{-\lambda_{j}}+\lambda_{j} \mathrm{e}^{-\lambda_{j}}+\mathrm{e}^{-\theta} \mathrm{e}^{-\lambda_{j}}\left(\mathrm{e}^{\lambda_{j} \mathrm{e}^{\theta}}-1-\lambda_{j} \mathrm{e}^{\theta}\right) \\
& =\mathrm{e}^{-\lambda_{j}}+\mathrm{e}^{-\theta} \mathrm{e}^{-\lambda_{j}}\left(\mathrm{e}^{\lambda_{j} \mathrm{e}^{\theta}}-1\right)=\mathrm{e}^{-\lambda_{j}}\left(1+\mathrm{e}^{-\theta}\left(\mathrm{e}^{\lambda_{j} \mathrm{e}^{\theta}}-1\right)\right)
\end{aligned}
$$


hence, if from now on we set

$$
\rho_{n}:=\frac{\mathrm{e}^{\lambda_{n} \mathrm{e}^{\theta}}-1}{\lambda_{n} \mathrm{e}^{\theta}}
$$

we have

$$
\begin{aligned}
\frac{1}{L(n)} \log P\left(\Delta_{n}>\delta\right) & \leq \frac{1}{L(n)} \sum_{j=1}^{n} \log \left(\mathrm{e}^{-\lambda_{j}}\left(1+\mathrm{e}^{-\theta}\left(\mathrm{e}^{\lambda_{j} \mathrm{e}^{\theta}}-1\right)\right)\right)-\theta \frac{\delta}{c} \\
& =-\frac{1}{L(n)} \sum_{j=1}^{n} \lambda_{j}+\frac{1}{L(n)} \sum_{j=1}^{n} \log \left(1+\lambda_{j} \rho_{j}\right)-\theta \frac{\delta}{c} .
\end{aligned}
$$

Then, by (3.4), we complete the proof if we show that, for all $\theta>0$,

$$
\lim _{n \rightarrow \infty} \frac{1}{L(n)} \sum_{j=1}^{n} \log \left(1+\lambda_{j} \rho_{j}\right)=\nu
$$

in fact, if (3.15) holds, we deduce that

$$
\limsup _{n \rightarrow \infty} \frac{1}{L(n)} \log P\left(\Delta_{n}>\delta\right) \leq-\theta \frac{\delta}{c}
$$

and we get (3.14) by letting $\theta$ go to infinity.

In order to prove (3.15) we remark that, since $\lambda_{n} \rightarrow 0$ (see Lem. 2.4), for all $\varepsilon \in(0,1)$ there exists $n_{0}$ such that

$$
1-\varepsilon<\rho_{n}<1+\varepsilon
$$

for all $n>n_{0}$. Hence

$$
\sum_{j=1}^{n} \log \left(1+\lambda_{j} \rho_{j}\right)=\sum_{j=1}^{n_{0}} \log \left(1+\lambda_{j} \rho_{j}\right)+\sum_{j=n_{0}+1}^{n} \log \left(1+\lambda_{j} \rho_{j}\right)
$$

and we can concentrate our attention on the second sum. Firstly, since $\log (1+z) \leq z$ for all $z>-1$, we have

$$
\sum_{j=n_{0}+1}^{n} \log \left(1+\lambda_{j} \rho_{j}\right) \leq \sum_{j=n_{0}+1}^{n} \log \left(1+\lambda_{j}(1+\varepsilon)\right) \leq(1+\varepsilon) \sum_{j=n_{0}+1}^{n} \lambda_{j}=(1+\varepsilon)\left(\sum_{j=1}^{n} \lambda_{j}-\sum_{j=1}^{n_{0}} \lambda_{j}\right),
$$

and we easily get the upper bound

$$
\limsup _{n \rightarrow \infty} \frac{1}{L(n)} \sum_{j=1}^{n} \log \left(1+\lambda_{j} \rho_{j}\right) \leq \nu
$$

by (3.4) and by the arbitrariness of $\varepsilon$. Now we take $n_{1}>n_{0}$ such that $\lambda_{n}<\varepsilon$ for all $n>n_{1}$; moreover, from the inequality $\log (1+z) \geq z-\frac{z^{2}}{2}$ (for $|z|$ small enough), we get

$$
\begin{aligned}
\sum_{j=n_{0}+1}^{n} \log \left(1+\lambda_{j} \rho_{j}\right) & \geq \sum_{j=n_{0}+1}^{n} \log \left(1+\lambda_{j}(1-\varepsilon)\right) \geq(1-\varepsilon) \sum_{j=n_{0}+1}^{n} \lambda_{j}-\frac{(1-\varepsilon)^{2}}{2} \sum_{j=n_{0}+1}^{n} \lambda_{j}^{2} \\
& \geq(1-\varepsilon) \sum_{j=n_{0}+1}^{n} \lambda_{j}-\frac{(1-\varepsilon)^{2}}{2} \sum_{j=n_{0}+1}^{n_{1}} \lambda_{j}^{2}-\frac{\varepsilon(1-\varepsilon)^{2}}{2} \sum_{j=n_{1}+1}^{n} \lambda_{j} ;
\end{aligned}
$$


then we obtain the matching lower bound

$$
\liminf _{n \rightarrow \infty} \frac{1}{L(n)} \sum_{j=1}^{n} \log \left(1+\lambda_{j} \rho_{j}\right) \geq \nu
$$

by (3.4) and by the arbitrariness of $\varepsilon$. In conclusion the limit relation (3.15) is checked and the proof of the proposition is complete.

\section{An EXAMPle OF INTEREST IN NUMBER THEORY}

In this section we apply Proposition 2.6 to a sequence of random variables $\left\{W_{n}: n \geq 1\right\}$ which appears in the probabilistic model for square-free numbers described in [5]. This sequence is given by suitable weighted means of independent Bernoulli distributed random variables $\left\{R_{n}: n \geq 1\right\}$, which is a particular case of Example 2.2. We also discuss the difficulties to obtain the LDP from the results in Section 3.

Throughout this section we denote by $2=p_{1}<p_{2}<p_{3}<\ldots$ the prime numbers, arranged in increasing order. We recall now the result proved in [5].

Theorem 4.1. Let $\left\{R_{n}: n \geq 1\right\}$ be independent random variables such that $R_{n} \stackrel{d}{\sim} \mathcal{B}\left(1 /\left(1+p_{n}\right)\right)$ for all $n \geq 1$. Let $\left\{W_{n}: n \geq 1\right\}$ be the sequence defined by $W_{n}:=\frac{1}{\log p_{n}} \sum_{k=1}^{n} R_{k} \log p_{k}$ for all $n \geq 1$. Then $\left\{W_{n}: n \geq 1\right\}$ converges weakly to $P_{W_{\infty}}=\mathcal{D}(1)$ as $n \rightarrow \infty$.

In the sequel we shall recover this result as a particular case of Proposition 2.6. This fact is interesting in that it shows that the property stated in Theorem 4.1 is not peculiar of square-free numbers, as one could guess looking at the proof given in [5] (based on deep properties of the prime numbers sequence). It is known that

$$
p_{n} \sim n \log n
$$

(see e.g. Thm. 8 in [18], p. 10) and

$$
\liminf _{n \rightarrow \infty} \frac{p_{n}-p_{n-1}}{\log p_{n}}=0 \text { and } \limsup _{n \rightarrow \infty} \frac{p_{n}-p_{n-1}}{\log p_{n}}=\infty
$$

(see [17] or [33] for the first one, and [37] for the second one). For all $n \geq 1$ we set

$$
\lambda_{n}:=\frac{1}{1+p_{n}} \text { and } \phi(n):=\log p_{n} .
$$

By (4.1), for all $\varepsilon \in(0,1)$ there exists $n_{0}$ such that

$$
(1-\varepsilon) n \log n \leq p_{n} \leq(1+\varepsilon) n \log n
$$

for all $n>n_{0}$. Then (1.2) holds because $\frac{\phi(n)}{\phi(n+1)} \rightarrow 1$ as it is easily proved. Moreover

$$
\frac{1}{\phi(n)} \sum_{k=n_{0}+1}^{n} \phi(k) \lambda_{k} \leq \frac{1}{\log [(1-\varepsilon) n \log n]} \sum_{k=n_{0}+1}^{n} \frac{\log [(1+\varepsilon) k \log k]}{1+p_{k}}
$$

and, by the arbitrariness of $\varepsilon$, we get

$$
\limsup _{n \rightarrow \infty} \frac{1}{\phi(n)} \sum_{k=n_{0}+1}^{n} \phi(k) \lambda_{k} \leq 1
$$


after some standard manipulations (in particular we use Cesaro's Theorem); so (1.5) holds because the matching lower bound can be derived similarly. Hence the weak convergence result stated in Proposition 2.6 is in force since we are dealing with Bernoulli distributed random variables $\left\{R_{n}: n \geq 1\right\}$ (and therefore this is a particular case of Example 2.2).

We conclude with a brief discussion on the difficulties to obtain the LDP from the results in Section 3. Firstly we cannot apply Proposition 3.2; in fact the function $\phi$ is neither concave nor convex because (see [10]) the inequalities $\phi(n+1)-\phi(n)>\phi(n)-\phi(n-1)$ and $\phi(n+1)-\phi(n)<\phi(n)-\phi(n-1)$ hold infinitely often. Moreover, by (4.1),

$$
\phi(n) \sim \log (n \log n) \sim \log (1+n)=: \phi^{*}(n)
$$

where $\phi^{*}$ is concave, but we cannot apply Proposition 3.4 either, because (3.6) is false. In fact, by the definition of $\phi^{*}$ in (4.3), (3.6) would mean that $\frac{\log \left(p_{n} / p_{n-1}\right)}{\log \left(1+\frac{1}{n}\right)} \rightarrow 1$ and, since $\frac{p_{n}}{p_{n-1}} \rightarrow 1$, by also taking into account (4.1) we would have

$$
1=\lim _{n \rightarrow \infty} n\left(\frac{p_{n}}{p_{n-1}}-1\right)=\lim _{n \rightarrow \infty} n \cdot \frac{p_{n}-p_{n-1}}{p_{n-1}}=\lim _{n \rightarrow \infty} \frac{p_{n}-p_{n-1}}{\log n}=\lim _{n \rightarrow \infty} \frac{p_{n}-p_{n-1}}{\log p_{n}}
$$

which contradicts (4.2) once more.

\section{A discussion on the Hellinger distance And a Further LDP}

We start by recalling the definition of the Hellinger distance $H^{2}\left[P_{1}, P_{2}\right]$ between two probability measures $P_{1}$ and $P_{2}$ on the same measurable space $\Omega$ (see e.g. Sect. 3.2 in [26]; see also Sect. 14.5 in [35]):

$$
H^{2}\left[P_{1}, P_{2}\right]:=\frac{1}{2} \int_{\Omega}\left(\sqrt{\frac{\mathrm{d} P_{1}}{\mathrm{~d} \mu}}-\sqrt{\frac{\mathrm{d} P_{2}}{\mathrm{~d} \mu}}\right)^{2} \mathrm{~d} \mu, \begin{aligned}
& \text { for any measure } \mu \text { such that } P_{1} \text { and } P_{2} \\
& \text { are absolutely continuous w.r.t. } \mu \text {. }
\end{aligned}
$$

Note that we also have $H^{2}\left[P_{1}, P_{2}\right]=1-A\left[P_{1}, P_{2}\right]$ where $A\left[P_{1}, P_{2}\right]:=\int_{\Omega} \sqrt{\frac{\mathrm{d} P_{1}}{\mathrm{~d} \mu} \frac{\mathrm{d} P_{2}}{\mathrm{~d} \mu}} \mathrm{d} \mu$ is the Hellinger affinity. We always have a choice for $\mu$, i.e. $\mu=P_{1}+P_{2}$.

In [16] (see Sect. 4.1) it was illustrated how the rate functions found there can be expressed in terms of the Hellinger distance with respect to the weak limit $P_{W_{\infty}}$ of the sequence $\left\{W_{n}: n \geq 1\right\}$ (the logarithmic means only are considered in [16]). More precisely, if $I$ is one of the rate functions of the LDPs in [16], then it was shown that

$$
(\mathbf{H}):\left\{\begin{array}{l}
\text { if } I(x)<\infty, \text { then we have the representation } \\
I(x):=-c \log \left(1-H^{2}\left[P(x), P_{W_{\infty}}\right]\right)=-c \log A\left[P(x), P_{W_{\infty}}\right], \\
\text { for some } c>0 \text { and some probability measure } P(x) \text { which depends on } x .
\end{array}\right.
$$

In this section we show that condition $(\mathbf{H})$ holds for the rate function $I$ in equation (1.6) with $P_{W_{\infty}}=\mathcal{D}(\nu)$. We also prove the LDP for a sequence $\left\{Z_{n}: n \geq 1\right\}$ defined by (1.1) for a suitable sequence $\left\{W_{n}: n \geq 1\right\}$ which converges weakly to $P_{W_{\infty}}=\mathcal{E}(\lambda)$ (for some $\lambda>0$ ), and we show that in this case the rate function does not meet condition $(\mathbf{H})$.

\subsection{On the rate function $I$ in equation (1.6)}

Notice that, for the examples in [16], the probability measure $P(x)$ in condition $(\mathbf{H})$ belongs to the exponential family generated by the weak limit $P_{W_{\infty}}$ of the sequence $\left\{W_{n}: n \geq 1\right\}$ (this feature was not highlighted in [16]). This has some analogy with what happens in Cramér Theorem (see e.g. Thm. 2.2.3 in [8]). In fact, if $\left\{X_{n}: n \geq 1\right\}$ 
is a sequence of i.i.d. real valued random variables with law $P_{X}$, the empirical means $\left\{\frac{1}{n} \sum_{k=1}^{n} X_{k}: n \geq 1\right\}$ satisfies the LDP with speed function $v_{n}=n$ and rate function $\Lambda^{*}$ defined by

$$
\Lambda^{*}(x):=\sup _{\theta \in \mathbb{R}}\{\theta x-\Lambda(\theta)\},
$$

where $\Lambda(\theta):=\log \mathbb{E}\left[\mathrm{e}^{\theta X_{1}}\right]$; then, if we denote the relative entropy (or Kullback-Leibler's divergence) between two probability measures by $\mathcal{H}(\cdot \mid \cdot)$, for all $x \in\left(\left\{y \in \mathbb{R}: \Lambda^{*}(y)<\infty\right\}\right)^{\circ}$ we have

$$
\Lambda^{*}(x)=\mathcal{H}\left(P_{X}^{\theta(x)} \mid P_{X}\right)
$$

where $\left\{P_{X}^{\theta}: \theta \in\{\eta \in \mathbb{R}: \Lambda(\eta)<\infty\}\right\}$ is the exponential family generated by $P_{X}$, and $\theta=\theta(x)$ is the solution of the equation $\Lambda^{\prime}(\theta)=x$ (this means that the expected value of the random variable $X_{1}$ under the law $P_{X}^{\theta(x)}$ is equal to $x$ ). We also recall that large deviation rate functions are often expressed in terms of the relative entropy; see e.g. the discussion in [36].

Then, by taking into account the weak convergence results in Section 2, we are led to consider the exponential family generated by $\mathcal{D}(\nu)$ for the examples in this paper. We set

$$
\kappa(s):=\nu \int_{0}^{s} \frac{\mathrm{e}^{y}-1}{y} \mathrm{~d} y,
$$

i.e. the function $\kappa$ is the logarithm of the moment generating function in (1.7); then the exponential family $\left\{Q_{s}: s \in \mathbb{R}\right\}$ generated by the Dickman's distribution $\mathcal{D}(\nu)=Q_{0}$ is formed by the family of probability measures with densities

$$
\frac{\mathrm{d} Q_{s}}{\mathrm{~d} Q_{0}}(x):=\mathrm{e}^{s x-\kappa(s)}
$$

with respect $Q_{0}$. In other words $Q_{s}$ is the Esscher-Cramér tilt of the distribution $Q_{0}$. Therefore the Hellinger's distance between $Q_{s}$ and $Q_{0}$ is

$$
H^{2}\left[Q_{s}, Q_{0}\right]:=\frac{1}{2} \int_{0}^{\infty}\left(\sqrt{\frac{\mathrm{d} Q_{s}}{\mathrm{~d} Q_{0}}(x)}-1\right)^{2} Q_{0}(\mathrm{~d} x),
$$

the Hellinger affinity is

$$
A\left[Q_{s}, Q_{0}\right]=\int_{0}^{\infty} \sqrt{\frac{\mathrm{d} Q_{s}}{\mathrm{~d} Q_{0}}(x)} Q_{0}(\mathrm{~d} x)=\int_{0}^{\infty} \mathrm{e}^{\frac{s}{2} x-\frac{\kappa(s)}{2}} Q_{0}(\mathrm{~d} x)=\mathrm{e}^{-\frac{\kappa(s)}{2}+\kappa\left(\frac{s}{2}\right)}
$$

and, by (5.1) and some computations, we obtain

$$
\begin{aligned}
-\log A\left[Q_{s}, Q_{0}\right] & =\frac{\kappa(s)}{2}-\kappa\left(\frac{s}{2}\right)=\frac{\nu}{2} \int_{0}^{s} \frac{\mathrm{e}^{y}-1}{y} \mathrm{~d} y-\nu \int_{0}^{\frac{s}{2}} \frac{\mathrm{e}^{y}-1}{y} \mathrm{~d} y \\
& =\frac{\nu}{2} \int_{0}^{s} \frac{\mathrm{e}^{y}-1}{y} \mathrm{~d} y-\nu \int_{0}^{s} \frac{\mathrm{e}^{y / 2}-1}{y / 2} \frac{\mathrm{d} y}{2}=\nu \int_{0}^{s} \frac{\left(\mathrm{e}^{y / 2}-1\right)^{2}}{2 y} \mathrm{~d} y .
\end{aligned}
$$

In conclusion condition $(\mathbf{H})$ holds for the rate function $I$ in equation (1.6) with $c=1$ and $P(x)=Q_{s(x)}$ (for all $x \geq 0)$, where $s(x)$ is a value such that

$$
\nu \int_{0}^{s(x)} \frac{\left(\mathrm{e}^{y / 2}-1\right)^{2}}{2 y} \mathrm{~d} y=x \log \frac{x}{\nu}-x+\nu .
$$

Note that in general we do not have a feasible expression of $s(x)$; however we know that $s(\nu)=0$. For instance, if we consider an increasing version of $s(\cdot)$, the range of values is $[s(0), \infty)$, and in particular we have $s(0) \simeq-16.593$ when $\nu=1$. Another version of $s(\cdot)$ could be decreasing in $[0, \nu)$ and increasing in $(\nu, \infty)$; in this case the range of values is $[0, \infty)$, and in particular we have $s(0) \simeq 2.492$ when $\nu=1$. 


\subsection{A further LDP}

Here we concentrate the attention on the following example. We define the random variables $\left\{W_{n}: n \geq 1\right\}$ in (1.1), and we study the LDP of $\left\{Z_{n}: n \geq 1\right\}$, in the case of logarithmic means.

Example 5.1. Let $\left\{X_{\lambda, n}: n \geq 1\right\}$ be independent random variables such that, for some $\lambda>0, X_{\lambda, n} \stackrel{d}{\sim} \mathcal{E}(\lambda)$ for all $n \geq 1$. We set $W_{n}:=n \min \left\{X_{\lambda, 1}, \ldots, X_{\lambda, n}\right\}$ (for all $n \geq 1$ ).

Proposition 5.2. Let $\left\{W_{n}: n \geq 1\right\}$ be as in Example 5.1, and set $\phi(n)=n$ for all $n \geq 1$. Then $\left\{Z_{n}: n \geq 1\right\}$ in (1.1) satisfies the LDP with speed function $v_{n}=L(n)$ and good rate function $I$ defined by $I(x):=(\sqrt{\lambda x}-1)^{2}$ for all $x \geq 0$ and $I(x):=\infty$ for all $x<0$.

Proof. We remark that

$$
\sum_{k=1}^{n} \frac{\phi(k)-\phi(k-1)}{\phi(k)} W_{k}=\sum_{k=1}^{n} \min \left\{X_{\lambda, 1}, \ldots, X_{\lambda, k}\right\} \quad(\text { for all } n \geq 1) .
$$

Then, since $L(n) \sim \log n$, the proposition will be proved if we show that

$$
\lim _{n \rightarrow \infty} \frac{1}{\log n} \log \mathbb{E}\left[\mathrm{e}^{\theta \sum_{k=1}^{n} \min \left\{X_{\lambda, 1}, \ldots, X_{\lambda, k}\right\}}\right]= \begin{cases}\frac{\theta / \lambda}{1-\theta / \lambda} & \text { for } \theta<\lambda \\ \infty & \text { for } \theta \geq \lambda\end{cases}
$$

for all $\theta \in \mathbb{R}$; in fact, by the Gärtner-Ellis's Theorem, the LDP holds with good rate function $I$ defined by

$$
I(x):=\sup _{\theta<\lambda}\left\{\theta x-\frac{\theta / \lambda}{1-\theta / \lambda}\right\}
$$

which coincides with the rate function $I$ in the statement of the proposition.

We remark that, for each fixed $n \geq 1$, the random variable $\sum_{k=1}^{n} \min \left\{X_{\lambda, 1}, \ldots, X_{\lambda, k}\right\}$ has the same distribution of $\frac{1}{\lambda} \sum_{k=1}^{n} \min \left\{X_{1,1}, \ldots, X_{1, k}\right\}$. Then, by equation (3.5) in [15], we have

$$
\frac{\log \mathbb{E}\left[\mathrm{e}^{\theta \sum_{k=1}^{n} \min \left\{X_{\lambda, 1}, \ldots, X_{\lambda, k}\right\}}\right]}{\log n}= \begin{cases}\frac{\log \prod_{k=1}^{n}\left(1+\frac{\theta / \lambda}{k(1-\theta / \lambda)}\right)}{\log n} & \text { if } \theta / \lambda<1 \\ \infty & \text { if } \theta / \lambda \geq 1\end{cases}
$$

(actually in that reference it was stated that $\theta / \lambda \in(0,1)$, but only the condition $\theta / \lambda<1$ is necessary). Finally the limit relation (5.2) follows noting that, if $\theta<\lambda$ (there is nothing to prove if $\theta \geq \lambda$ ), we have

$$
\begin{aligned}
\lim _{n \rightarrow \infty} \frac{\log \prod_{k=1}^{n}\left(1+\frac{\theta / \lambda}{k(1-\theta / \lambda)}\right)}{\log n} & =\lim _{n \rightarrow \infty} \frac{\sum_{k=1}^{n} \log \left(1+\frac{\theta / \lambda}{k(1-\theta / \lambda)}\right)}{\log n} \\
& =\lim _{n \rightarrow \infty} n \log \left(1+\frac{\theta / \lambda}{n(1-\theta / \lambda)}\right)=\frac{\theta / \lambda}{1-\theta / \lambda}
\end{aligned}
$$

by a standard application of Cesaro's Theorem.

We conclude with a discussion on condition $(\mathbf{H})$. It is known (and can be easily checked) that all the random variables $\left\{W_{n}: n \geq 1\right\}$ are $\mathcal{E}(\lambda)$ distributed; thus they converge weakly to $P_{W_{\infty}}=\mathcal{E}(\lambda)$ (as $\left.n \rightarrow \infty\right)$. Then, since $H^{2}\left[\mathcal{E}\left(\lambda_{1}\right), \mathcal{E}\left(\lambda_{2}\right)\right]=\frac{\left(\sqrt{\lambda_{1}}-\sqrt{\lambda_{2}}\right)^{2}}{\lambda_{1}+\lambda_{2}}$ (this equality can be checked by simple inspection), for the rate function $I$ in Proposition 5.2 we have

$$
I(x)=(\lambda x+1) H^{2}[\mathcal{E}(1 / x), \mathcal{E}(\lambda)](\text { for all } x \geq 0),
$$

and therefore condition $(\mathbf{H})$ fails. This could be explained noting that the sequence $\left\{W_{n}: n \geq 1\right\}$ in Example 5.1 is not a sequence of partial sums of random variables forming a triangular array as it happens for other examples in this paper, and for the examples in [16]. 
Acknowledgements. The authors wish to warmly thank two anonymous referees, whose remarks have led to a substantial improvement of the results of this paper. They also thank Olivier Ramaré for some useful discussions, and Barbara Pacchiarotti for the numerical values of $s(0)$ in Section 5.

\section{REFERENCES}

[1] R. Arratia and S. Tavaré, Independent processes approximations for random combinatorial structures. Adv. Math. 104 (1994) 90-154.

[2] M. Atlagh and M. Weber, Le théorème central limite presque sûr. Exp. Math. 18 (2000) 97-126.

[3] I. Berkes and E. Csáki, A universal result in almost sure central limit theory. Stoch. Process Appl. 94 (2001) $105-134$.

[4] G.A. Brosamler, An almost everywhere central limit theorem. Math. Proc. Camb. Philos Soc. 104 (1988) 561-574.

[5] F. Cellarosi and Y.G. Sinai, Non-Standard Limit Theorems in Number Theory. Prokhorov and Contemporary Probability Theory. Edited by A.N. Shiryaev, S.R.S. Varadhan and E.L. Presman. Springer, Heidelberg (2013) 197-213.

[6] S. Cheng, L. Peng and L. Qi, Almost sure convergence in extreme value theory. Math. Nachr. 190 (1998) 43-50.

[7] J.-M. De Koninck, I. Diouf and N. Doyon, On the truncated kernel function. J. Integer Seq. 15 (2012) Article 12.3 .2$.

[8] A. Dembo and O. Zeitouni, Large Deviations Techniques and Applications. 2nd edition. Springer, New York (1998).

[9] K. Dickman, On the frequency of numbers containing prime factors of a certain relative magnitude. Ark. Mat. Astron. Fys. 22 (1930) 1-14.

[10] P. Erdös and P. Turán, On some new questions on the distribution of prime numbers. Bull. Amer. Math. Soc. 54 (1948) $371-378$.

[11] I. Fahrner, An extension of the almost sure max-limit theorem. Stat. Probab. Lett. 49 (2000) 93-103.

[12] I. Fahrner and U. Stadtmüller, On almost sure max-limit theorems. Stat. Probab. Lett. 37 (1998) 229-236.

[13] A. Fisher, Convex-invariant means and a pathwise central limit theorem. Adv. Math. 63 (1987) 213-246.

[14] A. Fisher, A pathwise central limit theorem for random walk. Preprint (1989).

[15] M. Ghosh, G.J. Babu and N. Mukhopadhyay, Almost sure convergence of sums of maxima and minima of positive random variables. Z. Wahrsch. Verw. Gebiete 33 (1975) 49-54.

[16] R. Giuliano and C. Macci, Large deviation principles for sequences of logarithmically weighted means. J. Math. Anal. Appl. 378 (2011) 555-570.

[17] D.A. Goldston, J. Pintz and C.M. Ylldırım, Primes in tuples. I. Ann. Math. 170 (2009) 819-862.

[18] G.H. Hardy and E.M. Wright. An Introduction to the Theory of Numbers. 5th edition. The Clarendon Press, Oxford University Press, New York (1979).

[19] M.K. Heck, The principle of large deviations for the almost everywhere central limit theorem. Stoch. Process. Appl. 76 (1998) $61-75$.

[20] D. Hensley, The convolution powers of the Dickman function. J. London Math. Soc. 33 (1986) 395-406.

[21] A. Hildebrand and G. Tenenbaum, Integers without large prime factors. J. Théor. Nombres Bordeaux 5 (1993) $411-484$.

[22] S. Hörmann, On the universal a.s. central limit theorem. Acta Math. Hung. 116 (2007) 377-398.

[23] H.-K. Hwang and T.-H. Tsai, Quickselect and Dickman function. Combin. Probab. Comput. 11 (2002) 353-371.

[24] R. Kiesel and U. Stadtmüller, A large deviation principle for weighted sums of independent identically distributed random variables. J. Math. Anal. Appl. 251 (2000) 929-939.

[25] M.T. Lacey and W. Philipp, A note on the almost everywhere central limit theorem. Stat. Probab. Lett. 9 (1990) $201-205$.

[26] L. Le Cam and G.L. Yang, Asymptotics in Statistics. Some Basic Concepts. Springer-Verlag, New York (1990).

[27] P. Lévy. Sur certain processus stochastiques homogenes. Composition Math. 7 (1939) 283-339.

[28] M.A. Lifshits and E.S. Stankevich, On the large deviation principle for the almost sure CLT. Stat. Probab. Lett. 51 (2001) 263-267.

[29] M. Loève, Probability Theory I, 4th edition. Springer-Verlag, New York (1977).

[30] P. March and T. Seppäläinen, Large deviations from the almost everywhere central limit theorem. J. Theoret. Probab. 10 (1997) 935-965.

[31] A. Rouault, M. Yor and M. Zani, A large deviations principle related to the strong arc-sine law. J. Theoret. Probab. 15 (2002) 793-815.

[32] P. Schatte, On strong versions of the central limit theorem. Math. Nachr. 137 (1988) 249-256.

[33] K. Soundararajan, Small gaps between prime numbers: the work of Goldston-Pintz-Yıldırım. Bull. Amer. Math. Soc. (N.S.) 44 (2007) 1-18.

[34] G. Tenenbaum, Introduction to Analytic and Probabilistic Number Theory. Translated from the second French edition by C. B. Thomas. Cambridge University Press, Cambridge (1995).

[35] A.W. van der Vaart, Asymptotic Statistics. Cambridge University Press, New York (1998).

[36] S.R.S. Varadhan, Large deviations and entropy. Entropy. Edited by A. Greven, G. Keller and G. Warnecke. Princeton University Press (2003) 199-214.

[37] E. Westzynthius, Über die Verteilung der Zahlen die zu den $n$ ersten Primzahlen teilerfremd sind, Commun. Phys. Math. Helingsfors 5 (1931) 1-37. 